\title{
Sistemática del pez Petenia splendida (Perciformes: Cichlidae) en el lago Petén Itzá, Guatemala
}

\author{
Anaitté Méndez, María-Elena García \& Lourdes Lozano \\ Laboratorio de Ictiología, Facultad de Ciencias Biológicas, Universidad Autónoma de Nuevo León, Ave. Pedro de \\ Alba s/n cruz con Ave. Manuel L. Barragán San Nicolás de los Garza, Nuevo León, México; cele_anaitte@yahoo. \\ com.mx,mgarciar29@gmail.com,marlozan2006@gmail.com
}

Recibido 22-VII-2010. Corregido 10-I-2011. Aceptado 14-II-2011.

\begin{abstract}
Systematic of the fish Petenia splendida (Perciformes: Cichlidae) of Lake Petén Itzá, Guatemala. The Lake Petén Itzá is located in the multiple use zone of the Maya Biosphere Reserve. It belongs to the subtropical moist forest (warm) life zone, and has very important flora and fauna diversities. Among the fish species, the white fish (Petenia splendida) is of high commercial value. The main goal of the present work was to clarify the taxonomic position of the two forms of the white fish in Petén (Guatemala), and to compare it with the Usumacinta (Mexico) form, based on the collected material from 1978 and 2006 (Usumacinta), and collections made during 2008 and 2009 in the Lake Petén Itzá. Samples were obtained from 10 different locations in the lake. From the total of 57 fish specimens collected, 50 fish were selected ( 25 yellow form and 25 silver form); besides, 21 specimens from 10 locations of the Usumacinta river in Mexico, from the Fish Collection, FCB, UANL. A total of 36 morphometric and 16 meristic characteristics, were analyzed, and also coloration, habitat and fishing method. This study reports the P. splendida of the Lake Petén Itzá with the following characteristics: the yellow form at a depth of $0.5-1.5 \mathrm{~m}$ with dorsal fin XIV-(XV)-XVI/11-(12)-13, the silver form at a depth of 2-3m with dorsal fin XIV-(XV)-XVI/10-(12)-13, and the Usumacinta form with a dorsal fin XIV-(XV)$\mathrm{XV} / 12-(13)-13$. The discrimination analysis demonstrates a differentiation among the three analyzed populations. Some of the characteristics are the ocular diameter, the anal source-pelvic source distance and the dorsal source-anal source distance. The geographic area studied has a high diversity of fish fauna along the watershed, and it is possible to observe the anthropogenic damage, due to the overexploitation based the unapplicability of the existent regulation and the urbanization. If this situation continues it could jeopardize the survival of this emblematic specie. Rev. Biol. Trop. 59 (3): 1205-1216. Epub 2011 September 01.
\end{abstract}

Key words: Petenia splendida, systematic, Petén Itzá lake, cichlidae.

El Lago Petén Itzá forma parte de la Reserva de la Biosfera Maya, se encuentra ubicado en el departamento de Petén al norte de Guatemala, Centro América (16 $54^{\prime} 00^{\prime \prime} \mathrm{N}-89^{\circ} 41^{\prime} 41^{\prime \prime}$ W). El Petén cuenta con una vasta variedad de especies de plantas y animales de gran importancia para el país por ser especies nativas, dentro de ellas podemos mencionar al pez blanco Petenia splendida, nativo de la familia de los ciclidos; ampliamente distribuido en ríos, lagunas y en el lago Petén Itzá, es la especie de mayor interés de pesca para los pobladores, debido a su alto precio comercial y la calidad de su carne. Los pescadores artesanales han detectado tres formas de $P$. splendida (Günther 1862): roja, amarilla y plateada. Actualmente sólo quedan las últimas dos ya que la roja no se ha localizado. Con este estudio sistemático se propone definir el estatus taxonómico que le corresponde a las tres formas de Petenia reportadas y con ello ampliar la plataforma de antecedentes de la misma.

Las publicaciones referentes a $P$. splendida para el área son insuficientes o muy antiguas, 
en otros casos la información no es puntual. Tomando en cuenta que el Petén es una área predominantemente cubierta de selva, con una exuberante vegetación tropical y de alta importancia con respecto a su biodiversidad a nivel del istmo Centro Americano, la inclusión de líneas de investigación alternas que incluyan las especies endémicas del lugar es de vital importancia para el desarrollo sustentable del departamento.

Entre la información sobre la especie tenemos la descripción original realizada por Günther (1862) para el Lago Petén Itzá, en donde se determinó que posee un cuerpo con escamas ctenoideas, aleta dorsal XV/12 y aleta anal V/10. En el sureste de México y Belice se encontraron datos representativos de la especie en los trabajos de: Velasco-Colín (1976), quien menciona que para Chiapas tiene una aleta dorsal XV-XVI/12-13 y anal V/8-10; DomínguezCisneros \& Rodiles-Hernández (1998) en una guía de peces en el río Lacanjá, México, indicaron que esta especie se caracteriza porque la boca generalmente sobresale la mandíbula y que tiene una aleta dorsal XV/13 y anal V/10. Schmitter-Soto (1998) apunta que en diversas localidades de Quintana Roo, la boca de esta especie puede extenderse casi la longitud de la cabeza y que tienen una aleta dorsal XIVXVI/12-13 y anal IV-VI/8-10. Wessel en 1993 para diversas áreas de Belice, menciona la presencia de dos coloraciones de $P$. splendida, la oro-marrón y la oro-roja para el área natural.

Es importante conocer la situación taxonómica del complejo Petenia para con ello determinar si existe alguna variación morfométrica y/o merística importante que nos permita distinguir las diferentes formas, lo que servirá de apoyo para los estudios de su biología, de tal forma que pueda utilizarse en acuicultura, igualmente en la reglamentación y regulación de su explotación en pesquerías para que esta sea sustentable.

\section{MATERIALES Y MÉTODOS}

Ellago Petén Itzá es el tercero de importancia en Guatemala por su extensión correspondiente a $99 \mathrm{~km}^{2}$, se encuentra a $110 \mathrm{~m}$. s.n.m., su origen geológico es tectónico y por levantamiento de la plataforma marina, debido a las características geomorfológicas de su ubicación no posee drenaje superficial y la densidad de corrientes superficiales en sus alrededores es baja. El lago es la principal fuente de agua y alimento para las cabeceras municipales que se ubican a su alrededor: Flores, San Benito, San Andrés y San José (Castañeda-Salguero 1995). CanoAlvarado en el 2002 indica que el lago es la principal fuente de agua y alimento para las cabeceras municipales que se ubican a su alrededor, mencionadas anteriormente.

Entre 2008 y 2009 se realizaron dos viajes de recolección al Lago Petén Itzá (1654'00”-

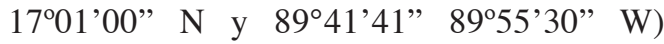
con una duración promedio de 40 días cada uno. Los ejemplares fueron obtenidos en 10 sitios de muestreo en el lago Petén Itzá y 10 en el Usumacinta, México (Fig. 1): 1. La Base Flores: $16^{\circ} 55^{\prime} 5^{\prime \prime} \mathrm{N}-89^{\circ} 51^{\prime} 27^{\prime \prime} \mathrm{W}$; 2. Petencito: $16^{\circ} 55^{\prime} 32^{\prime \prime} \mathrm{N}-89^{\circ} 52^{\prime} 5^{\prime \prime} \mathrm{W} ; 3$. Arrozal: $16^{\circ} 55^{\prime} 46^{\prime \prime} \mathrm{N}-89^{\circ} 52^{\prime} 40^{\prime \prime} \mathrm{W} ; 4$. Esplendido: 16 $55^{\prime} 30^{\prime \prime} \mathrm{N}-89^{\circ} 53^{\prime} 31^{\prime \prime} \mathrm{W} ; 5$. Salón de Flores: 1655'39" N-8953'39” W; 6. San Andrés: $16^{\circ} 57^{\prime} 56^{\prime \prime} \mathrm{N}-89^{\circ} 54^{\prime} 49^{\prime \prime} \mathrm{W}$; 7. San José: $16^{\circ} 58^{\prime} 40^{\prime \prime} \mathrm{N}-89^{\circ} 54^{\prime} 16^{\prime \prime} \mathrm{W} ; 8$. Paraíso Maya: $17^{\circ} 00^{\prime} 31^{\prime \prime} \mathrm{N}-89^{\circ} 52^{\prime} 17^{\prime \prime} \mathrm{W}$; 9. Jobompiche: $16^{\circ} 59^{\prime} 35^{\prime \prime} \mathrm{N}-89^{\circ} 43^{\prime} 28^{\prime \prime}$ w y 10. El Remate: $16^{\circ} 59^{\prime} 18^{\prime \prime} \mathrm{N}-89^{\circ} 41^{\prime} 45^{\prime \prime} \mathrm{W}$. Los ejemplares del Usumacinta de México se obtuvieron de las siguientes 10 localidades: 11. Catazajá: $17^{\circ} 43$ '52" N-92 0'49" W; 12. San Pedro: $17^{\circ} 47^{\prime} 13^{\prime \prime}$ N-91 ${ }^{\circ} 9^{\prime} 8^{\prime \prime}$ W; 13. Usumacinta: $16^{\circ} 37^{\prime} 22^{\prime \prime} \mathrm{N}-90^{\circ} 39^{\prime} 43^{\prime \prime} \mathrm{W}$; 14. Lacanja: $16^{\circ} 24^{\prime} 14^{\prime \prime} \mathrm{N}-90^{\circ} 47^{\prime} 52^{\prime \prime} \mathrm{W} ; 15$. Tzendales: $16^{\circ} 17^{\prime} 46^{\prime \prime} \mathrm{N}-90^{\circ} 53^{\prime} 07^{\prime \prime} \mathrm{W} ; 16$. Negro: $16^{\circ} 11^{\prime} 30^{\prime \prime} \mathrm{N}-90^{\circ} 49^{\prime} 49^{\prime \prime} \mathrm{W}$; 17 . Caracol: 1609'27" N-9053’37” W; 18. LacantúnUsumacinta: $16^{\circ} 06^{\prime} 35^{\prime \prime} \mathrm{N}-90^{\circ} 56^{\prime} 23$ " W; 19. Chajulillo: $16^{\circ} 06^{\prime} 09^{\prime \prime}$ N-99 $57^{\prime} 09^{\prime \prime}$ W y 20. Chajul: 1606'11" N-9057'22" W.

Ambos cuerpos de agua son de importancia para cada país, debido a que confluyen en formas de manejo y utilización del recurso, ya sea económicamente en el caso del departamento de Petén, Guatemala, y Usumacinta, 


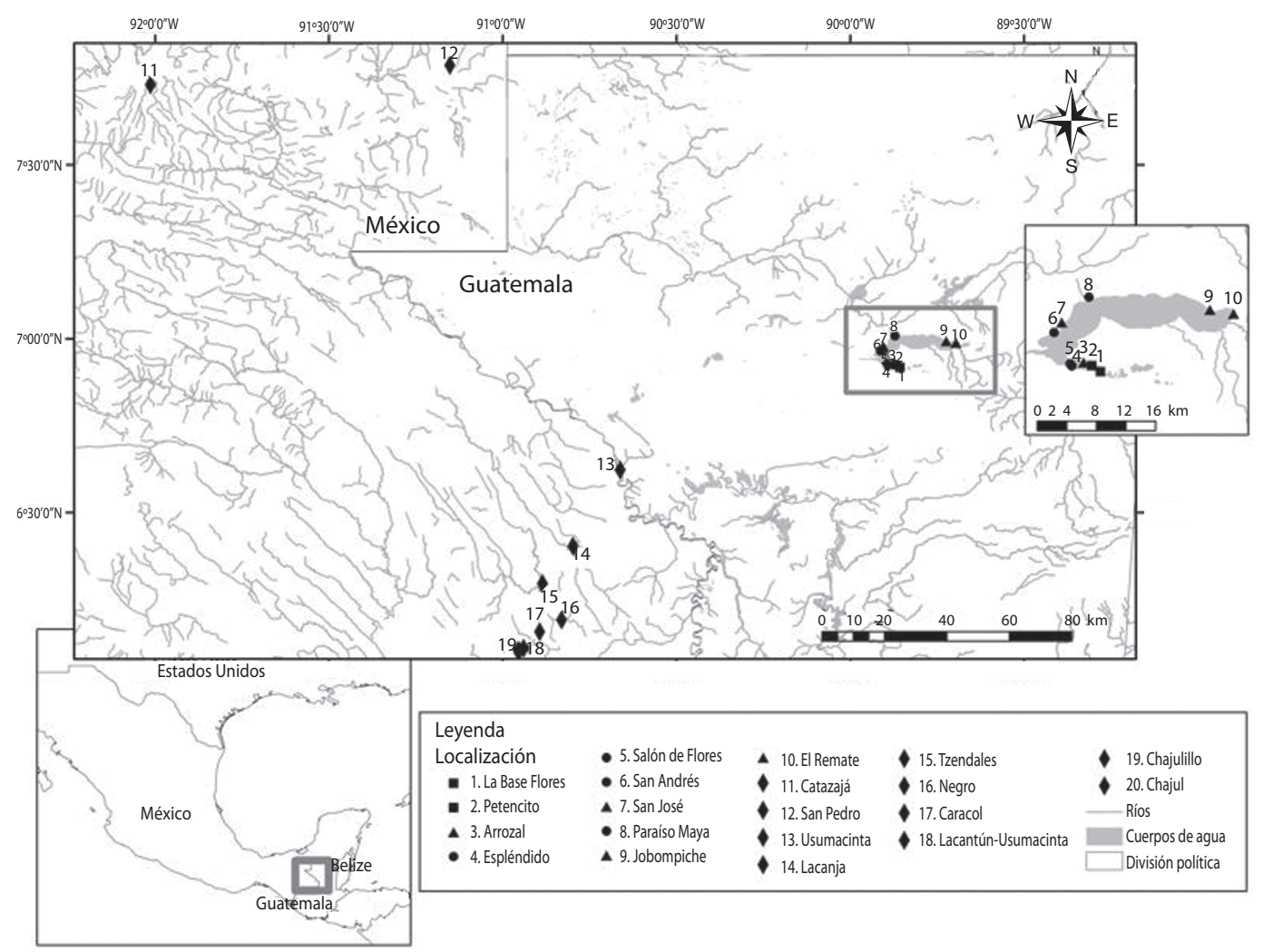

Fig. 1. Localidades de recolección en Lago Petén Itzá, Guatemala y Río Usumacinta, México. (Diseño: Biol. Hidalgo Rodríguez Vela).

Fig. 1. Collection localities in Lake Petén Itzá, Guatemala and Usumacinta River, México.

México, en vista de que sirve como frontera natural entre ambos países.

Para la captura de los peces en el lago Petén Itzá se utilizo chinchorro de 3/16 de luz de malla por $3 \mathrm{~m}$ de largo y $1.80 \mathrm{~m}$ de alto, y redes experimentales de 1/2, 1, 1 1/2 y 2 pulgadas de luz de malla, atarrayas de $3 \frac{1}{2}$ pies con luz de malla de $3 / 8$ ' de luz y redes de cuchara. En total se capturaron 50 ejemplares, los cuales fueron fijados en formol al $10 \%$, durante siete días, posteriormente lavados en agua corriente por $24 \mathrm{hr}$ y preservados en alcohol isopropílico $50 \%$.

Las siguientes características fisicoquímicas del agua se tomaron in situ: temperatura del agua, oxígeno disuelto, dureza con un aparato HACH CEL/890 AWL, pH con HACH SENSION1 (Cuadro 1), además de las características ecológicas de los hábitats de las formas amarilla y plateada.

La determinación del estatus taxonómico se basó en Álvarez del Villar (1970), VelascoColín (1976), Domínguez-Cisneros \& RodilesHernández (1998) y Miller et al. (2005); se realizaron biometrías a 71 ejemplares, 21 del Río Usumacinta, que forman parte de la Colección Ictiológica (UANL) y 50 del Lago Petén, según Lozano-Vilano (1991) y García-Ramírez (1997). El material se encuentra depositado en la Colección Ictiológica de la Facultad de Ciencias Biológicas, Universidad Autónoma de Nuevo León (UANL). 
CUADRO 1

Calidad del agua en los lugares de recolecta de las dos formas de P. splendida en el lago Petén Itzá, Petén, Guatemala

TABLE 1

Water quality parameters obtained during the collection of the two forms of P. splendida from Lake Petén Itzá, Petén, Guatemala

\begin{tabular}{|c|c|c|c|c|c|c|}
\hline Población & Lugar & $\mathrm{OD}(\mathrm{mg} / \mathrm{L})$ & Temperatura $\left({ }^{\circ} \mathrm{C}\right)$ & $\mathrm{pH}$ & $\begin{array}{c}\text { Dureza mg/L } \\
\text { de } \mathrm{CaCO}_{3}\end{array}$ & $\begin{array}{l}\text { Temperatura } \\
\text { ambiente }\left({ }^{\circ} \mathrm{C}\right)\end{array}$ \\
\hline \multirow[t]{2}{*}{ Plateada } & Salón de Flores & 7.7 & 28.4 & 8.1 & 583 & 24.1 \\
\hline & Esplendido & 8.5 & 28.6 & 8.2 & 588 & 23.9 \\
\hline \multirow[t]{2}{*}{ Ambas } & Petencito & 7.7 & 28.9 & 8.2 & 590 & 23.5 \\
\hline & La Base & 8 & 24.9 & 8.2 & 582 & 17.6 \\
\hline Amarilla & Arrozal & 7.9 & 25.3 & 8.2 & 578 & 17.3 \\
\hline \multirow[t]{3}{*}{ Amarilla } & Jobompiche & 7.6 & 30.0 & 8.5 & 624 & 22.8 \\
\hline & El Remate & 7.5 & 29.4 & 8.4 & 680 & 22.1 \\
\hline & San José & 7.8 & 30.7 & 8.5 & 681 & 27.5 \\
\hline \multirow[t]{2}{*}{ Plateada } & San Andrés & 7.7 & 30.5 & 8.5 & 688 & 33.3 \\
\hline & Paraíso Maya & 7.6 & 30.4 & 8.4 & 688 & 27.8 \\
\hline
\end{tabular}

Las biometrías se realizaron bajo el microscopio estereoscópico, se tomaron 36 características morfométricas en milímetros con un vernier de puntas Helios 2 TRUR de cuadrante, hasta la décima del milímetro más cercana, según el método de Hubbs \& Lagler (1974) las medidas tomadas fueron: longitud patrón, longitud de la aleta dorsal deprimida, longitud cefálica, longitud de la aleta anal deprimida, distancia predorsal, longitud base de la aleta pectoral, distancia postdorsal, base postdorsal-base postanal, altura máxima, base postdorsal-abanico hipural, altura mínima, longitud del pedúnculo caudal, diámetro ocular, origen dorsal-origen pectoral, rostro-origen anal, origen anal-abanico hipural, diámetro de la pupila, origen anal-origen pélvico, distancia preorbital, longitud base de la aleta dorsal, rostro-origen pectoral, origen dorsal-base postanal, distancia postorbital, longitud base de la aleta anal, rostro-origen pélvico, anchura de la boca, distancia interorbital, longitud base de la aleta pélvica, pélvica-origen pectoral, longitud de la mandíbula, pélvica-base postdorsal, longitud de la maxila, longitud de la aleta pectoral, origen dorsal-origen anal, longitud de la aleta pélvica, base postdorsal-origen anal y 16 características merísticas radios dorsales, escamas de la línea lateral, espinas dorsales, escamas alrededor del cuerpo, radios anales, escamas transversal dorsal-pélvica, espinas anales, escamas transversal dorsal-anal, radios caudales, escamas circumpedunculares, radios pectorales, branquiespinas arco superior e inferior, radios pélvicos, espinas pélvicas y manchas opérculo-pedúnculo (Fig. 2).

Los datos biométricos obtenidos fueron sometidos a un análisis de función discriminante (ADF) con el programa SPSS, versión 15.0 mediante el análisis discriminante se obtuvo el scattergram para las funciones canónicas discriminantes 1 y 2 , el análisis de clasificación y la función canónica con el método de mahalanobis.

Para la presentación del material examinado se siguió a Leviton et al. (1985) y quedó de

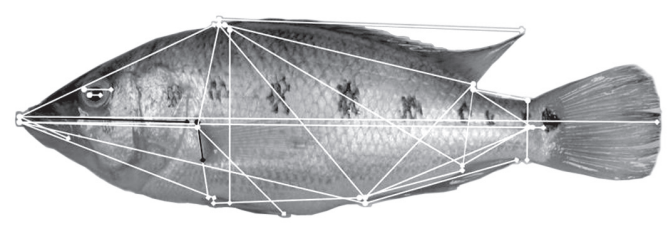

Fig. 2. Características morfométricas de $P$. splendida.

Fig. 2. Morphometric characteristics in P. splendida. 
la siguiente forma: Cuenca, País: siglas UANL con el número de catalogo, entre paréntesis el número de ejemplares: LP mínima y máxima en $\mathrm{mm}$, localidad, recolectores y fecha de recolecta, como se cita a continuación:

Lago Peten Itzá, Guatemala: UANL 19215 (5:179.20-210.55) Salón de Flores, C.A. Méndez-G., J.A. Morales-V. y M.J. Tut-P., diciembre 21, 2008; UANL 19216 (8:174.45-208.30) Esplendido, C.A. Méndez-G., J.A. Morales-V. y M.J. Tut-P., diciembre 25, 2008; UANL 19217 (7:144.80-229.80) Petencito, C.A. Méndez-G., J.A. Morales-V. y M.J. Tut-P., diciembre 29, 2008; UANL 19218 (6:164.38-225.72) La Base, C.A. Méndez-G., J.A. Morales-V. y M.J. Tut-P., enero 2, 2009; UANL 19219 (9:149.50-219.71) El Arrozal, C.A. MéndezG., J.A. Morales-V. y M.J. Tut-P., enero 6, 2009; UANL 19220 (3:149.44-184.25) Jobompiche, C.A. Méndez-G., J.A. Morales-V. y M.J. Tut-P., junio 24, 2009; UANL 19221 (2:153.45-192.01) El Remate, C.A. MéndezG., J.A. Morales-V. y M.J. Tut-P., junio 28, 2009; UANL 19222 (3:156.16-177.36) San José, C.A. Méndez-G., J.A. Morales-V. y M.J. Tut-P., julio 1, 2009; UANL 19223 (3:157.58200.15) San Andrés, C.A. Méndez-G., J.A. Morales-V. y M.J. Tut-P., julio 5, 2009; UANL 19224 (4:168.75-186.55) Paraíso Maya, C.A. Méndez-G., J.A. Morales-V. y M.J. Tut-P., julio 9, 2009.

Usumacinta, México: 2917 (1:197.2) San Pedro; Colectores: S. Contreras-B., J.M. Torres-A. y grupo de 60 de Biología II, Febrero 14, 1978; UANL 6368 (1:103.3) Laguna de Catazaja, M.L. Lozano-V., S. Contreras-B. y A.J. Contreras-B., Noviembre 6, 1985; UANL 10048 (4: 87.1-179.7) Lacanja, S. Contreras-B., M.L. Lozano-V. y A.J. Contreras-B., Octubre 17, 1988. UANL 10853 (1:218.7) Usumacinta, M.L. Lozano-V. y A.J. Contreras-B., Mayo 8, 1980; UANL 15713 (1:163.4) Chajul, M. L. Lozano-V., M.E. García-R., A.J. Contreras-B. y personal Estación Chajul, Agosto 2, 2004; UANL 15772 (1:122.4) Lacantún-Usumacinta, M.L. Lozano-V., M.E. García-R., A.J. Contreras-B. y personal Estación Chajul, Agosto 25, 2004; UANL 15987 (1:133.8) Chajul, M.L.
Lozano-V., M.E. García-R., A.J. Contreras-B. y personal Estación Chajul, Febrero 9, 2005; UANL 16616 (1:113.8) Lacantún-Usumacinta, M.L. Lozano-V., M.E. García-R., A.J. Contreras-B. y personal Estación Chajul, Junio 5, 2005. UANL 16634 (1:136.5) Chajul, M.L. Lozano-V., M.E. García-R., A.J. Contreras-B. y personal Estación Chajul, Junio 6, 2005; UANL 16835 (1:87.3) Negro, M.L. Lozano-V., M.E. García-R., A.J. Contreras-B. y personal Estación Chajul, Septiembre 13, 2005; UANL 16849 (1:110.0) Lacantún-Usumacinta, M.L. Lozano-V., M.E. García-R., A.J. Contreras-B. y personal Estación Chajul, Septiembre 13, 2005; UANL 16871 (1:113.7) Tzendales, M.L. Lozano-V., M.E. García-R., A.J. ContrerasB. y personal Estación Chajul, Septiembre 14, 2005. UANL 16979 (1:222.2) LacantúnUsumacinta, M.L. Lozano-V., M.E. García-R., A.J. Contreras-B. y personal Estación Chajul, Diciembre 9, 2005. UANL 17041 (1:109.5) Chajulillo, M.L. Lozano-V., M.E. García-R., A.J. Contreras-B. y personal Estación Chajul, Diciembre 11, 2005; UANL17178 (1:229.6) Lacantún-Usumacinta, M.L. Lozano-V., M.E. García-R., A.J. Contreras-B. y personal Estación Chajul, Febrero 28, 2006. UANL 17262 (2: 126.9-129.4) Lacantún-Usumacinta, M.L. Lozano-V., M.E. García-R., A.J. Contreras-B. y personal Estación Chajul, Marzo 2, 2006; UANL 17564 (1:127.0) Caracol, M.L. LozanoV., M.E. García-R., A.J. Contreras-B. y personal Estación Chajul, Junio 13, 2006.

\section{RESULTADOS}

Los peces recolectados en el lago Petén Itzá fueron 50 ejemplares del grupo P. splendida, los cuales se examinaron y se encontraron dos formas diferentes (forma plateada y forma amarilla) (Fig. 3), la forma roja no fue encontrada en las localidades muestreadas, se amplió la búsqueda a otras áreas del lago Petén Itzá, pero de acuerdo a los pescadores, el área ha sido sobreexplotada de tal manera que las poblaciones han declinado drásticamente, sobretodo la forma roja. 

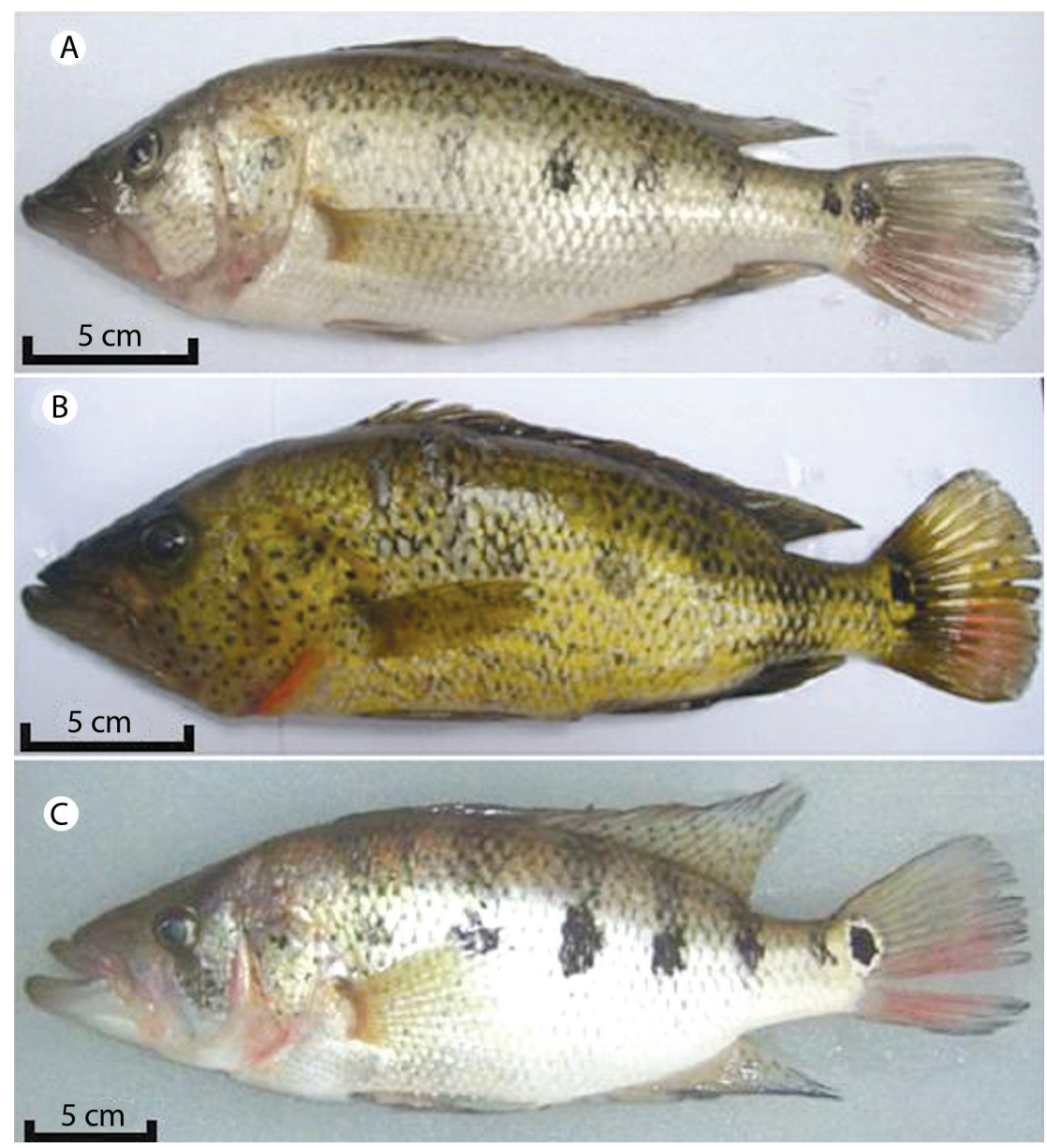

Fig. 3. Formas de P. splendida: A. Forma plateada, Lago Petén Itzá, B. Forma amarilla, Lago Petén Itzá, C. Forma Usumacinta, México.

Fig. 3. Forms of P. splendida: A. Silver form, Lake Petén Itzá, B. Yellow form, Lake Petén Itzá, C. Usumacinta, México form.

\section{Forma Plateada}

La caracterización de la forma plateada fue la siguiente: cuerpo alargado y comprimido, más robusto en las hembras y más alargado en los machos, con una coloración plateada más oscura en el dorso y más clara en el vientre, en pocos organismos se observan pequeños puntos negros sobre las escamas, en la parte media del cuerpo se presentan de siete a nueve manchas negras que van desde el opérculo hasta el pedúnculo caudal, seis individuos presentaron pequeños puntos negros dispersos por la aleta caudal, 20 organismos presentan pequeños puntos negros dispersos en la aleta dorsal, un individuo presento pequeños puntos negros dispersos en la aleta pectoral y solo seis individuos entre los radios de la caudal, en ocasiones pueden presentar coloración roja en la aleta caudal.

La longitud de la espina del premaxilar es menor a la longitud cefálica con una proporción de 1.2 veces, la altura máxima del cuerpo 2.9 a 3.2 veces en la longitud patrón y 
la longitud cefálica 2.5 a 2.8 veces en la longitud patrón, diámetro del ojo de 4.1 a 6.1 en la longitud cefálica.

El hocico se presenta comprimido y triangular, boca terminal excesivamente protráctil, la mandíbula inferior sobresale de la maxila, presenta dientes viliformes, escamas ctenoideas. La aleta dorsal presenta de 14 a 16 espinas largas y fuertes, y de 10-13 radios, la aleta anal cuatro a seis espinas fuertes y siete a diez radios, la aleta caudal presenta 14-16 radios, las aletas pélvicas presentan generalmente una espina, raramente dos y cinco radios, las aletas pectorales presentan de 11-13 radios; se observan cuatro branquiespinas en la parte superior y nueve a 11 en la parte inferior, las escamas en la línea lateral con 34 a 44 (Cuadro 2), (Fig. 3).

Esta variedad prefiere un tipo de fondo lodoso o pedregoso que no esté asociado con plantas acuáticas, se encuentran a una profundidad que varía entre los 2-3m, a una distancia de la orilla de hasta $147 \mathrm{~m}$.

\section{Forma Amarilla}

Se caracteriza por tener cuerpo alargado y comprimido, más robusto en las hembras y más alargado en los machos, de coloración amarilla con pequeños puntos negros sobre las escamas y el opérculo, en la parte media del cuerpo se presentan de siete a nueve manchas negras que van desde el opérculo hasta el pedúnculo caudal, pueden presentarse puntos dispersos sobre los radios de la aleta caudal, dorsal, anal y un individuo los presentó en la pectoral, ocasionalmente pueden presentar coloración roja en la aleta caudal.

La longitud de la espina del premaxilar es menor a la longitud cefálica con una proporción de 1.1 veces, la altura máxima del cuerpo 2.6 a 3.4 veces en la longitud patrón y

\section{CUADRO 2}

Moda, mínimo y máximo de las características merísticas de las poblaciones amarilla y plateada del lago Petén Itzá, Petén, Guatemala y la forma del Usumacinta, México

TABLE 2

Mode, minimum and maximum from the meristic characteristics of silver and yellow population collected in Lake Petén Itzá, Petén, Guatemala and the form of the Usumacinta, México

\begin{tabular}{lccccccccc}
\multicolumn{1}{c}{ Característica } & \multicolumn{3}{c}{ Plateada } & \multicolumn{4}{c}{ Amarilla } & \multicolumn{3}{c}{ Usumacinta } \\
& Xmin & Mo. & Xmax & Xmin & Mo. & Xmax & Xmin & Mo. & Xmax \\
Radios dorsales & 10 & $(12)$ & 13 & 11 & $(12)$ & 13 & 12 & $(13)$ & 13 \\
Espinas dorsales & 14 & $(15)$ & 16 & 14 & $(15)$ & 16 & 14 & $(15)$ & 15 \\
Radios caudales & 14 & $(16)$ & 16 & 16 & $(16)$ & 17 & 15 & $(16)$ & 16 \\
Radios anales & 7 & $(9)$ & 10 & 8 & $(9)$ & 10 & 8 & $(9)$ & 10 \\
Espinas anales & 4 & $(5)$ & 6 & 5 & $(5)$ & 6 & 4 & $(5)$ & 5 \\
Radios pectorales & 11 & $(13)$ & 13 & 11 & $(13)$ & 13 & 12 & $(13)$ & 14 \\
Radios pélvicos & 5 & $(5)$ & 5 & 5 & $(5)$ & 5 & 5 & $(5)$ & 5 \\
Espinas pélvicas & 1 & $(1)$ & 2 & 1 & $(1)$ & 1 & 1 & $(1)$ & 1 \\
Escamas de la línea lateral & 34 & $(41)$ & 44 & 34 & $(37)$ & 45 & 34 & $(38)$ & 39 \\
Escamas alrededor del cuerpo & 49 & $(52)$ & 57 & 45 & $(50)$ & 56 & 50 & $(53)$ & 56 \\
Escamas transversales dorsal-pélvica & 20 & $(23)$ & 26 & 19 & $(23)$ & 25 & 21 & $(22)$ & 25 \\
Escamas transversales dorsal-anal & 9 & $(9)$ & 13 & 9 & $(10)$ & 10 & 9 & $(10)$ & 10 \\
Escamas circumpedunculares & 19 & $(20)$ & 25 & 19 & $(23)$ & 27 & 20 & $(23)$ & 25 \\
Branquiespinas superiores & 4 & $(4)$ & 4 & 3 & $(4)$ & 4 & 3 & $(4)$ & 4 \\
Branquiespinas inferiores & 9 & $(10)$ & 11 & 9 & $(10)$ & 11 & 10 & $(10)$ & 12 \\
Manchas del opérculo al pedúnculo caudal & 7 & $(8)$ & 9 & 7 & $(8)$ & 9 & 8 & $(8)$ & 8
\end{tabular}


la longitud cefálica 2.4 a 2.7 veces en la longitud patrón, diámetro del ojo de 5.0 a 6.9 en la longitud cefálica.

Cabeza con hocico comprimido y triangular, boca terminal excesivamente protráctil, la mandíbula inferior sobresale de la maxila, los dientes son viliformes; escamas ctenoideas; la aleta dorsal presenta 14-16 espinas largas y fuertes, y 11-13 radios; la aleta anal tiene cinco a seis espinas fuertes y de ocho a 10 radios, la aleta caudal presenta 16-17 radios, las aletas pélvicas con una espina y cinco radios, las aletas pectorales poseen 11-13 radios; con tres a cuatro branquiespinas en la parte superior del primer arco y nueve a 11 en la parte inferior, con 34 a 35 escamas en la línea lateral (Cuadro 2), (Fig. 3).

Esta variedad prefiere un tipo de fondo asociado con plantas acuáticas como Vallisneria sp., Potamogeton sp., Lentibularia sp., Cabomba sp. y Najas sp., se encuentran a una profundidad que varía entre los $0.5-1.5 \mathrm{~m}$, a una distancia de la orilla menor a los $15 \mathrm{~m}$.

\section{Forma Usumacinta}

La forma del Usumacinta, México, presenta un solo patrón de coloración, además muestran un cuerpo alargado y comprimido, más alargado en los machos y más robusto en las hembras; en la parte media de los costados tiene machas de color negro que van desde el opérculo hasta el pedúnculo caudal, puede poseer una coloración roja sobre la aleta caudal y el opérculo; hocico comprimido y triangular, boca terminal excesivamente protráctil, la mandíbula inferior sobresale de la maxila, los dientes son viliformes.

La longitud de la espina del premaxilar es menor a la longitud cefálica con una proporción de 1.3 veces, la altura máxima del cuerpo 2.7 a 3.2 veces en la longitud patrón y la longitud cefálica 2.5 a 2.9 veces en la longitud patrón, diámetro del ojo de 4.3 a 6.5 en la longitud cefálica (Cuadro 3).

La aleta dorsal presenta 14-15 espinas largas y fuertes, y 12-13 radios, la aleta anal

CUADRO 3

Media, mínimo y máximo de las proporciones morfométricas con mayor divergencia de las poblaciones plateada y amarilla del lago Petén Itzá, Petén Guatemala y del río Usumacinta, México

TABLE 3

Mean, minimum and maximum from the proportions of the morphometric characteristics with greater divergence of silver and yellow population collected in Lake Petén Itzá, Petén, Guatemala and Usumacinta River, Mexico

\begin{tabular}{|c|c|c|c|c|c|c|c|c|c|c|}
\hline \multicolumn{2}{|c|}{ Proporciones } & \multirow{2}{*}{$\begin{array}{c}\mathrm{LP} / \mathrm{LC} \\
2.5\end{array}$} & \multirow{2}{*}{$\begin{array}{c}\text { LP/DP1 } \\
2.4\end{array}$} & \multirow{2}{*}{$\begin{array}{c}\mathrm{LP} / \mathrm{DA} \\
2.31\end{array}$} & \multirow{2}{*}{$\begin{array}{c}\text { LP/PDA } \\
3.1\end{array}$} & \multirow{2}{*}{$\begin{array}{c}\mathrm{LP} / \mathrm{PDH} \\
6.1\end{array}$} & \multirow{2}{*}{$\begin{array}{c}\text { LP/AMA } \\
2.9\end{array}$} & \multirow{2}{*}{$\begin{array}{c}\text { LP/AMI } \\
8.7\end{array}$} & \multirow{2}{*}{$\begin{array}{c}\mathrm{LC} / \mathrm{AB} \\
3.0\end{array}$} & \multirow{2}{*}{$\begin{array}{c}\mathrm{LC} / \mathrm{DO} \\
4.1\end{array}$} \\
\hline & MIN & & & & & & & & & \\
\hline \multirow[t]{3}{*}{ Amarilla } & MEDIA & 2.6 & 2.5 & 2.5 & 3.4 & 6.7 & 3.1 & 9.3 & 4.1 & 5.1 \\
\hline & MAX & 2.8 & 2.6 & 2.5 & 3.5 & 7.3 & 3.2 & 9.8 & 4.8 & 6.1 \\
\hline & MIN & 2.4 & 2.3 & 2.21 & 2.3 & 5.9 & 2.6 & 8.2 & 3.8 & 5.0 \\
\hline \multirow[t]{3}{*}{ Plateada } & MEDIA & 2.6 & 2.6 & 2.4 & 3.3 & 6.6 & 3.1 & 9.2 & 4.2 & 5.7 \\
\hline & MAX & 2.7 & 2.7 & 2.6 & 3.7 & 7.7 & 3.4 & 10.1 & 4.6 & 6.9 \\
\hline & MIN & 2.5 & 2.3 & 2.17 & 2.9 & 4.9 & 2.7 & 6.5 & 3.3 & 4.3 \\
\hline \multirow[t]{2}{*}{ Usumacinta } & MEDIA & 2.6 & 2.5 & 2.3 & 3.2 & 6.6 & 3.0 & 8.5 & 4.0 & 5.0 \\
\hline & MAX & 2.9 & 2.8 & 2.4 & 3.6 & 7.8 & 3.2 & 9.4 & 4.7 & 6.5 \\
\hline
\end{tabular}

LP: longitud patrón, LC: longitud cefálica, DP1: distancia pre-dorsal, DA: origen dorsal-origen anal, PDA: base postdorsalorigen anal, PDH: base postdorsal-abanico hipural, AMA: altura máxima, AMI: altura mínima, AB: anchura de la boca, DO: diámetro ocular. 
tiene cuatro a cinco espinas fuertes y ocho a 10 radios, la aleta caudal posee 16 radios, las aletas pélvicas con una espina y cinco radios, las aletas pectorales tienen 12-14 radios; poseen de tres a cuatro branquiespinas en la parte superior y de 10 a 12 en la parte inferior, con 34 a 49 escamas en la línea lateral (Cuadro 2), (Fig. 3).

En cuanto a la merística se obtuvo poca divergencia, cabe destacar que las manchas del opérculo al pedúnculo caudal en el caso de la forma del Usumacinta en todos los ejemplares fue de ocho, mientras que en la forma amarilla y plateada varia de siete a nueve manchas. Se analizaron además las proporciones de las características morfométricas (Cuadro 3) y aunque no se presentó marcada diferencia se pudo observar algunos rangos disímiles entre las formas analizadas.

La calidad fisicoquímica del agua (Cuadro 1) en los diferentes sitios de muestreo se observó para la forma plateada de P. splendida el oxígeno disuelto de $(7.6-8.5 \mathrm{mg} / \mathrm{l})$, la temperatura $\left(24.9-30.5^{\circ} \mathrm{C}\right)$, la dureza $(582-688 \mathrm{mg}$ de $\mathrm{CaCO} 3)$ y el $\mathrm{pH}(8.1-8.5)$; y la forma amarilla el oxígeno disuelto de $(7.5-8 \mathrm{mg} / \mathrm{l})$, la temperatura $\left(24.9-30.7^{\circ} \mathrm{C}\right)$, la dureza $(578-681 \mathrm{mg}$ de $\mathrm{CaCO} 3)$ y el pH (8.2-8.5).

Se realizo un análisis biométrico de 36 variables morfométricas de 71 ejemplares de 20 localidades de Petenia, 25 de las localidades $1,2,3,7,9$ y 10; 25 de las localidades $1,2,4$, 5,6 , y 8 ; y 21 de las localidades 11-20; estas fueron comparadas y analizadas mediante el análisis discriminante. Los valores de Wilk's Lambda variaron de 0.053 a 0.782 , lo cual indica una fuerte discriminación entre las localidades comparadas. Las funciones canónicas 1 (F1) y 2 (F2) explican $76.2 \%$ y $23.8 \%$, respectivamente de la varianza $(\mathrm{p}<0.000)$. El resultado de clasificación muestra que el $100 \%$ de los ejemplares examinados fueron correctamente agrupados. Los caracteres morfométricos más significativos en la discriminación fueron para la F1: origen anal-origen pélvico 1.195; diámetro ocular 1.101; base postdorsal-origen anal -0.118; origen dorsal-origen anal -1.163 y origen dorsal-origen pectoral -1.422. (Fig. 4).

\section{DISCUSIÓN}

De acuerdo con los resultados obtenidos en este trabajo sobre características taxonómicas de $P$. splendida, observamos que Günther (1862) describió esta especie con cuerpo comprimido alargado, escamas ctenoideas 41 en la línea lateral, dientes cónicos, boca protráctil, aletas: dorsal XV/12, anal V/10, localidad de recolección Lago Peten Itzá, Guatemala, pero a diferencia de la presente investigación no reportó la existencia de los tres colores que se sabe existen en el área, aunque una de ellas, la roja no se le ha vuelto a ver, en el caso de las otras dos coloraciones, la plateada y la amarilla se obtuvo que los ejemplares evaluados tienen dientes viliformes, Günther por su parte aunque menciona algunos aspectos de coloración, no menciona las manchas características de la especie que van del opérculo al pedúnculo caudal, estas fueron indicadas en la descripción de la especie encontradas durante el desarrollo de la fase de campo de la presente investigación.

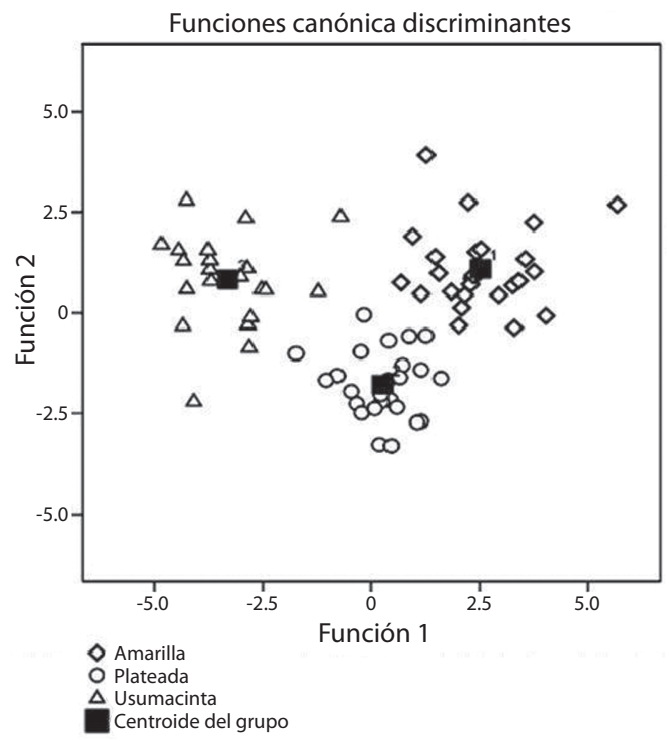

Fig. 4. Distribución de las funciones canónicas discriminantes 1 y 2 de las formas de P. splendida (amarilla y plateada) del Lago Petén Itzá y del Usumacinta, México. Fig. 4. Distribution of canonical discriminant functions 1 and 2 of forms P. splendida (amarilla y plateada) of Lake Petén Itzá and the Usumacinta, Mexico. 
Los trabajos que se describen a continuación fueron realizados en diferentes localidades de México y únicamente reportan coloración amarilla en todas las localidades. VelascoColín (1976) mencionó a Petenia con cuerpo ligeramente alargado especialmente en los machos, escamas en la línea lateral de 38 a 45 , coloración dorada en el dorso más oscura y en el vientre más clara con nueve manchas oscuras a lo largo del cuerpo del opérculo a la base de la aleta caudal, dientes viliformes, boca grade y protráctil, espinas y radios: dorsal $\mathrm{XV}-\mathrm{XVI} / 12-13$, anal V/8-10, recolectados en varias localidades de Chiapas, México; para la presente investigación se reporta el número de escamas en la línea lateral con un rango menor, al igual que el número de manchas oscuras a lo largo del cuerpo que también es menor.

Domínguez-Cisneros \& Rodiles-Hernández (1998) describen una $P$. splendida de cuerpo ligeramente alargado, escamas en línea lateral 38-40, coloración amarilla con siete manchas negras, boca protráctil donde sobresale la mandíbula, la longitud de la espina del premaxilar puede ser igual o mayor a la longitud cefálica, presenta de 10 a 12 branquiespinas en la rama inferior, la altura máxima del cuerpo tres veces en la longitud patrón y la longitud cefálica 2.8 veces en la longitud patrón, diámetro del ojo de cinco a seis en la longitud cefálica, espinas y radios: dorsal XV/13, anal V/10 recolectadas en las localidades del río Lacanjá, México. Comparados los datos anteriores con el presenta trabajo se reporta un dato menor en la longitud de la espina del premaxilar en las tres coloraciones, el rango en número de branquiespinas es igual con la coloración del Usumacinta, sin embargo la coloración amarilla y plateada presentan un rango menor, en cuanto a las proporciones altura máxima del cuerpo/longitud patrón y longitud cefálica/longitud patrón se presentan rangos menores, en el ancho del cuerpo se encontró un rango mayor, finalmente se observaron rangos más variables en las espinas y radios dorsales con esto se amplía la información de la especie.

Schmitter-Soto (1998) indicó qué P. splendida posee un cuerpo amarillento con siete bandas transversales y manchas redondeadas negras en el pedúnculo caudal y frente a las pectorales, aletas verticales con puntos negros en la base y entre los radios, con 34-45 escamas en la línea lateral, boca protráctil que puede extenderse casi la longitud de la cabeza, espinas y radios: dorsal XIV-XVI/12-13 y anal IV-VI/8-10, pectoral 14-15, altura máxima $31-39 \%$ en la longitud patrón y longitud cefálica $34-39 \%$ en la longitud patrón, recolectados en localidades de Quintana Roo; el comparativo con el presente trabajo indica que no se observaron bandas transversales en ningún ejemplar al contrario se observan las manchas negras que van del opérculo al pedúnculo ocular, no se encontraron puntos negros en la base de las aletas de ningún ejemplar, y únicamente los ejemplares de coloración plateada y amarilla presentan puntos negros entre los radios de la aleta dorsal y caudal, las proporciones altura máxima/longitud patrón y longitud cefálica/ longitud patrón presentaron rangos similares, así mismo los rangos de espinas y radios dorsales y anales presentan rangos similares.

Wessel (1993) menciona dos coloraciones de P. splendida en la laguna White Water de Belice: oro-marrón y oro-rojo, a diferencia de las obtenidas del lago Petén Itzá, donde igualmente muestran dos coloraciones pero difieren ya que son una amarilla y la otra plateada, con características ecológicas diferentes para cada forma tales como la profundidad, tipo de fondo, plantas acuáticas, y distancia de la orilla. Mientras que las del río Usumacinta, México presentó una sola coloración amarilla.

La merística comparativa se encuentra de esta forma (la plateado) con: pélvica I-II 15 , caudal 14-16 y pectoral 11-13; la coloración amarilla con: pélvica I/5, caudal 16-17 y pectoral 11-13; y la Petenia del Usumacinta de México con: pélvica $\mathrm{I} / 5$, caudal 16 y pectoral 12-14.

El análisis discriminante muestra la separación en las tres poblaciones, la aparición de las diferencias fenológicas de las formas descritas se deben a las diferentes condiciones ecológicas y de hábitats encontrados esto nos da un indicativo de que pudiera estar iniciando 
un proceso de especiación entre las formas debido al aislamiento espacial, puesto que la distribución de las dos coloraciones del lago Petén Itzá, está fuertemente asociada con sus requerimientos de sustrato, con esto se concluye que la forma amarilla prefiere áreas de menor profundidad con presencia de plantas acuáticas cercanas a la orilla, mientras que la forma plateada habita a mayores profundidades con fondos lodosos o pedregosos. Esto permitió identificar las zonas de muestreo con mayor facilidad.

El lago Petén Itzá ha recibido fuertes impactos causados por la sobreexplotación del agua, debido al aumento de la agricultura, de la misma forma en los últimos años se ha visto una fuerte degradación de la cuenca y del lago en sí, es por ello que la $P$. splendida está incluida en el Listado de Especies Amenazadas (LEAs) del Consejo Nacional de Áreas Protegidas-CONAP- de Guatemala en el índice y tres, siendo una especie endémica que se encuentra amenazada por explotación y pérdida del hábitat natural, sin embargo el estado de su población permite su uso y manejo regulado con fines científicos y comerciales controlados. La contaminación de la cuenca y del lago Petén Itzá, el poco o nulo control de la actividad pesquera artesanal y la presión de captura sobre la especie, son las principales causas de la disminución de la población natural de la especie.

Con los datos obtenidos se observa que la diferencia entre las formas se empieza a presentar no únicamente a nivel de coloración si no también a nivel morfométrico, sin embargo se necesitan estudios genéticos que puedan determinar la línea evolutiva de las formas. La biometría que se presenta en este trabajo proporciona una plataforma más amplia al momento de la clasificación de los ejemplares de este género, ayuda al conocimiento de la especie y demuestra la importancia de la conservación de los hábitats naturales.

\section{AGRADECIMIENTOS}

Agradecemos de manera especial a Alejandro Ovalle por su ayuda en el laboratorio y a Tere Guadarrama por su apoyo en copiado de material bibliográfico, consejos y amistad. A Hidalgo Rodríguez Vela por el diseño del mapa de las localidades de estudio. El apoyo brindado en los viajes de recolección a: M. José Tut P. y a Julio Morales por su colaboración activa durante los cruceros de captura y actividades de campo. La ayuda brindada por la Organización de los Estados Americanos por financiar los viajes de recolección. A la Universidad de San Carlos de Guatemala por la colaboración con materiales y equipo. La recolección de peces se realizó en convenio con el proyecto de investigación de la Petenia splendida con la supervisión y apoyo de Manuel Ixquiac Cabrera.

\section{RESUMEN}

El Lago Petén Itzá se ubica en la zona de usos múltiples de la Reserva de la Biosfera Maya, entre sus especies ícticas encontramos al pez blanco (Petenia splendida) de alto valor comercial. El propósito del presente trabajo es aclarar la posición taxonómica de las dos formas de pez blanco de Petén y compararlas con la forma del Usumacinta. Entre 2008 y 2009 se recolectaron 25 ejemplares de la forma amarilla y 25 de la plateada en 10 localidades del lago Petén Itzá y 21 en la cuenca del Usumacinta durante 1978 y 2006; se revisaron 36 características morfométricas y 16 merísticas, así como coloración, hábitat y forma de pesca. La $P$. splendida del Lago presenta las siguientes características: la forma amarilla se encuentra a una profundidad entre 0.5 y $1.5 \mathrm{~m}$, tiene aleta dorsal XIV-(XV)$\mathrm{XVI} / 11-(12)-13$; la plateada esta a profundidades entre 2-3m, presenta aleta dorsal XIV-(XV)-XVI/10-(12)-13, mientras que la forma del Usumacinta posee aleta dorsal XIV-(XV)-XV/12-(13)-13. El análisis discriminante muestra una diferenciación entre las tres poblaciones analizadas aunque no es determinante. La fauna de peces de la cuenca del área de estudio presenta alta diversidad, se observa daño antropogénico producto de la sobreexplotación, por la falta de aplicación de la reglamentación existente y la urbanización.

Palabras clave: Petenia, splendida, lago Petén Itzá, sistemática, pez blanco, Usumacinta.

\section{REFERENCIAS}

Álvarez del Villar, J. 1970. Peces mexicanos (claves). Instituto Nacional de Investigaciones Biológico Pesqueras. Secretaría de Industria y Comercio. México D.F., México. 
Cano-Alvarado, M.F. 2002. Perfil ambiental del Petén. Ministerio de Agricultura, Ganadería y Alimentación de Guatemala, Petén, Guatemala.

Castañeda-Salguero, C. 1995. Sistemas lacustres de Guatemala. Universitaria, Guatemala.

Domínguez-Cisneros, S. \& R. Rodiles-Hernández. 1998. Guía de peces del río Lacanjá, selva Lacandona, Chiapas, México. Colegio de la Frontera Sur, Chiapas, México.

García-Ramírez, M.E. 1997. Revisión sistemática del complejo Fundulus grandis (Pisces: Fundulidae) del Noreste de México. Tesis de Maestría, Universidad Autónoma de Nuevo León, Nuevo León, México.

Günter, A. 1862. Catalogue of the fishes in the British Museum. British Museum, Londres, Inglaterra.

Hubbs, C.L. \& K.F. Lagler. 1974. Fishes of the Great Lakes Region. University of Michigan, Michigan, EEUU.

Leviton, A.E., R.H. Gibbs Jr., E. Heal \& C.E. Dawson. 1985. Standards in herpetology and ichthyology: Part
I. Standard symbolic codes for institutional resource collections in herpetology and ichthyology. Copeia 3: $802-832$.

Lozano-Vilano, M.L. 1991. Sistemática, evolución y zoogeografía del complejo alvarezi de los peces cyprinodontidos del género Cyprinodon en el sur de Nuevo León, Mexico. Tesis de Maestría, Universidad Autónoma de Nuevo León, Nuevo León, México.

Miller, R.R., W.L. Minckley \& S.M. Norris. 2005. Freshwater fishes of México. University of Chicago, Chicago, EEUU.

Schmitter-Soto, J.J. 1998. Catálogo de los peces continentales de Quintana Roo. Colegio de la Frontera Sur, Chiapas, México.

Velasco-Colín, R. 1976. Los peces de agua dulce del Estado de Chiapas. Gobierno del Estado de Tuxtla Gutiérrez, Chiapas, México.

Wessel, R. 1993. In search of the Red Bay Snook. Buntbarsche Bull. 157: 9-13. 